H. P. Fujita

Nagoya Math. J.

Vol. 124 (1991), 145-155

\title{
MANSFIELD AND SOLOVAY TYPE RESULTS ON COVERING PLANE SETS BY LINES
}

\author{
HIROSHI P. FUJITA
}

F. van Engelen, K. Kunen, and A. W. Miller proved, in [EKM], that for every analytic $\left(\boldsymbol{\Sigma}_{1}^{1}\right)$ set $A$ on the plane, either $A$ can be covered by a countable family of lines or else there is a perfect subset $P$ of $A$ such that no three points of $P$ are collinear. In this paper, we present some generalizations of their result. In particular, a question which was raised by van Engelen et al. in the last paragraph of [EKM] is answered (see Section 3).

We first consider generalizations to $\kappa$-Suslin sets and $\Sigma_{2}^{1}$ sets on the plane (Section 1). A lightface refinement of the result of van Engelen et al. is also examined (Section 2).

\section{$\S 1$. Covering a Suslin set}

In this section, we prove the following theorem, which is a direct generalization of the theorem of van Engelen et al.

Theorem 1. Let $A \subseteq \mathbb{R}^{2}$ be a $k$-Suslin set and let $T^{0}$ be the tree associated to a $\kappa$-semiscale on $A$. Then either $A$ can be covered by an $L\left[T^{0}\right]$ definable family of lines with size at most $\kappa$, or else there is a perfect subset of $A$ with no three collinear points.

See Chapter 2 of [Mo] for definitions of " $k$-Suslin", " $k$-semiscale," etc. We may view Baire's space ${ }^{\omega} \omega$ of irrationals as a subset of the plane $\mathbb{R}^{2}$ since ${ }^{\omega} \omega$ is homeomorphic to the set of points in $\mathbb{R}^{2}$ with irrational coordinates. So, $\mathbb{R}^{2}-{ }^{\omega} \omega$ is the union of an arithmetically definable family of countably many lines.

For a given $\kappa$-Suslin set $A$, let $T^{0}$ be the tree on $\omega \times \kappa$ associated to a $\kappa$-semiscale on $A$. We may suppose, without loss of generality, that $A \subseteq{ }^{\omega} \omega$ and $A$ is the projection of $T^{0}$ where the projection $\mathrm{p}[T]$ of a tree

Received January 8, 1991. 
$T$ is defined, as in [Mo], by the following formula.

$$
\mathrm{p}[T]=\{x \mid(\exists f)(\forall n)[\langle x| n, f\lceil n\rangle \in T]\} .
$$

For a tree $T$ on $\omega \times \kappa$, we define the derivative $T^{\prime}$ of $T$ as

$$
T^{\prime}=\{\langle u, v\rangle \in T \mid \mathrm{p}[T(u, v)] \text { is not contained in a line }\} \text {. }
$$

Here, $T(u, v)$ is a set of sequences $\left\langle u^{\prime}, v^{\prime}\right\rangle$ in $T$ which are comparable with $\langle u, v\rangle$. Given $T^{0}$ we define the transfinite sequence $\left\langle T^{\alpha} \mid \alpha \in \operatorname{Ord}\right\rangle$ of trees on $\omega \times \kappa$ inductively as follows:

$$
\left\{\begin{array}{l}
T^{\alpha+1}=\left(T^{\alpha}\right)^{\prime} \\
T^{\lambda}=\bigcap_{\alpha<\lambda} T^{\alpha}, \quad \text { if } \lambda \text { is a limit ordinal. }
\end{array}\right.
$$

The method of our proof of Theorem 1 is a mixture of the method used by van Engelen et al. and some effective considerations as in Mansfield's perfect set theorem (8G.2 and 8G.13 of [Mo]).

Lemma 1. Let $T$ be a tree on $\omega \times \kappa$. If $\mathrm{p}[T(u, v)]$ is contained in a line, then there is an $L[T]$-definable line which covers $\mathrm{p}[T(u, v)]$.

Proof. If $\mathrm{p}[T(u, v)]$ is empty or a singleton, it follows that the set is in $L[T]$ and so there surely exists such an $L[T]$-definable line containing it. On the other hand, if $\mathrm{p}[T(u, v)]$ has at least two elements, then there exists a unique line $l$ which contains it. Hence

$$
\begin{aligned}
x \in l & \Longleftrightarrow(\exists y, z)[y, z \in \mathrm{p}[T(u, v)] \& y \neq z \& x \in \overline{y z}] \\
& \Longleftrightarrow(\forall y, z)[y, z \in \mathrm{p}[T(u, v)] \& y \neq z \Longleftrightarrow x \in \overline{y z}] .
\end{aligned}
$$

Here, $\overline{x y}$ denotes the line passing through points $x$ and $y$. Since the set $\mathrm{p}[T(u, v)]$ is $\Sigma_{1}$-definable over $L[T]$, the above equivalences give a $\Delta_{1}$ definition of $l$ over $L[T]$.

Lemma 2. The transfinite sequence $\left\langle T^{\alpha}\right| \alpha \in$ Ord $\rangle$ is $L\left[T^{0}\right]$-definable. In particular, we have $T^{\alpha} \in L\left[T^{0}\right]$ for all $\alpha$.

Proof. Since $L\left[T^{0}\right]$ is an inner model of set theory, it suffices to show that the derivation $T \mapsto T^{\prime}$ of trees is an absolute operation for inner models. To see this, note that

$$
\begin{aligned}
\langle u, v\rangle \in T^{\prime} & \Longleftrightarrow \mathrm{p}[T(u, v)] \text { is not contained in a line } \\
& \Longleftrightarrow \text { No } L[T] \text {-definable line can contain } \mathrm{p}[T(u, v)] \\
& \Longleftrightarrow L[T] \vDash " \mathrm{p}[T(u, v)] \text { is not contained in a line." }
\end{aligned}
$$


These equivalences follow from the fact that collinearity is an absolute notion.

Lemma 3. Let $T$ be a tree on $\omega \times \kappa$. Suppose that for each $\langle u, v\rangle \in T$, $\mathrm{p}[T(u, v)]$ is not contained in a line. Then $\mathrm{p}[T]$ contains a perfect set with no three collinear points.

To simplify notations, let $A(u, v)$ denote the set $\mathrm{p}[T(u, v)]$. We say $y \in{ }^{\omega} \kappa$ witnesses that $x \in A(u, v)$ if

$$
u \subset x, \quad v \subset y,
$$

and

$$
(\forall n)[\langle x \uparrow n, y\lceil n\rangle \in T] .
$$

The lemma is proved via the following two claims.

ClaIm 1. Suppose that no line meets more than two of the

$$
A\left(u_{0}, v_{0}\right), A\left(u_{1}, v_{1}\right), \cdots, A\left(u_{n}, v_{n}\right) .
$$

Then there are two extensions $\left\langle u_{0}^{p}, v_{0}^{p}\right\rangle(p=0,1)$ of $\left\langle u_{0}, v_{0}\right\rangle$ and extensions $\left\langle\tilde{u}_{i}, \tilde{v}_{i}\right\rangle$ of $\left\langle u_{i}, v_{i}\right\rangle(i=1, \cdots, n)$ respectively such that no line meets more than two of the

$$
A\left(u_{0}^{0}, v_{0}^{0}\right), A\left(u_{0}^{1}, v_{0}^{1}\right), A\left(\tilde{u}_{1}, \tilde{v}_{1}\right), \cdots, A\left(\tilde{u}_{n}, \tilde{v}_{n}\right) .
$$

Proof. Since $A\left(u_{0}, v_{0}\right)$ is not contained in any line, it has more than one elements. Let $x_{0}^{0}$ and $x_{0}^{1}$ be two distinct elements of $A\left(u_{0}, v_{0}\right)$ and let $y_{0}^{0}$ and $y_{0}^{1}$ witness that $x_{0}^{0} \in A\left(u_{0}, v_{0}\right)$ and $x_{0}^{1} \in A\left(u_{0}, u_{0}\right)$ respectively. Pick $x_{i} \in A\left(u_{i}, v_{i}\right)-\overline{x_{0}^{0} x_{0}^{1}}$ and let $y_{i}$ witness that $x_{i} \in A\left(u_{i}, v_{i}\right)$ for $i=1, \cdots, n$. Then by the assumption on $A\left(u_{i}, v_{i}\right)(i=0, \cdots, n)$, no line can contain three of the $x_{0}^{0}, x_{0}^{1}, x_{1}, \cdots, x_{n}$. It follows that there are open neighbourhoods $U_{0}^{0}, U_{0}^{1}, U_{1}, \cdots, U_{n}$ of $x_{0}^{0}, x_{0}^{1}, x_{1}, \cdots, x_{n}$ respectively such that no line meets more than two of them. Choose pairs of sequences

$$
\left\langle u_{0}^{0}, v_{0}^{0}\right\rangle,\left\langle u_{0}^{1}, v_{0}^{1}\right\rangle,\left\langle\tilde{u}_{1}, \tilde{v}_{1}\right\rangle, \cdots,\left\langle\tilde{u}_{n}, \tilde{v}_{n}\right\rangle
$$

to be initial segments of

$$
\left\langle x_{0}^{0}, y_{0}^{0}\right\rangle,\left\langle x_{0}^{1}, y_{0}^{1}\right\rangle,\left\langle x_{1}, y_{1}\right\rangle, \cdots,\left\langle x_{n}, y_{n}\right\rangle
$$

respectively, which are long enough to make it sure that

$$
A\left(u_{0}^{0}, v_{0}^{0}\right) \subseteq U_{0}^{0}, \quad A\left(u_{0}^{1}, v_{0}^{1}\right) \subseteq U_{0}^{1},
$$


and

$$
A\left(\tilde{u}_{1}, \tilde{v}_{1}\right) \subseteq U_{1}, \cdots, A\left(\tilde{u}_{n}, \tilde{v}_{n}\right) \subseteq U_{n} .
$$

The conclusion of the claim follows immediately.

Claim 2. Suppose that no line meets more than two of the

$$
A\left(u_{0}, v_{0}\right), A\left(u_{1}, v_{1}\right), \cdots, A\left(u_{n}, v_{n}\right) .
$$

Then, for each $\left\langle u_{i}, v_{i}\right\rangle$, there are two extensions $\left(u_{i}^{0}, v_{i}^{0}\right\rangle$ and $\left\langle u_{i}^{1}, v_{i}^{1}\right\rangle$ such that no line meets more than two of the

$$
A\left(u_{0}^{0}, v_{0}^{0}\right), A\left(u_{0}^{1}, v_{0}^{1}\right), \cdots, A\left(u_{n}^{0}, v_{n}^{0}\right), A\left(u_{n}^{1}, v_{n}^{1}\right) .
$$

Proof. Use Claim 1 repeatedly.

Proof of Lemma 3. Using Claim 2, we can assign for each finite sequence $\sigma \epsilon^{<\omega} 2$ of zeros and ones, a pair of sequences $\langle u(\sigma), v(\sigma)\rangle$ in $T$ such that whenever $\sigma_{0}, \sigma_{1}$ and $\sigma_{2}$ are incomparable no line meets all of the $A\left(u\left(\sigma_{i}\right), v\left(\sigma_{i}\right)\right)(i=0,1,2)$.

For each $t \in{ }^{\omega} 2$, define

$$
F(t)=\bigcup_{n \in \omega} u(t \uparrow n) .
$$

Then $F$ is a continuous function on ${ }^{\omega} 2$ into ${ }^{\omega} \omega$, and for each $t \in{ }^{\omega} 2$, we have $F(t) \in A$ as witnessed by $\bigcup_{n \in \omega} v(t \uparrow n)$. Moreover, if $r, s, t \in{ }^{\omega_{2}} 2$ are distinct then $F(r), F(s)$, and $F(t)$ are not collinear.

Hence, the image of $F$ is a perfect subset of $A$ with no three collinear points.

We are now ready to give a proof of Theorem 1 .

Proof of Theorem 1. Let $A$ and $\left\langle T^{\alpha}\right| \alpha \in$ Ord $\rangle$ be as above. Since

$$
T^{0} \supseteq T^{1} \supseteq \cdots \supseteq T^{a} \supseteq \cdots
$$

and since $T^{0}$ has size at most $\kappa$, there must be an ordinal $\delta<\kappa^{+}$such that

$$
T^{\delta}=T^{\delta+1}=\bigcap_{\alpha \in \text { Ord }} T^{\alpha}=\bigcap_{\alpha<\delta} T^{\alpha} .
$$

We should consider two possible cases:

Case 1: $T^{\delta}=\emptyset$.

In this case, $A$ can be covered by a family $\left\{l(u, v) \mid\langle u, v\rangle \in T^{0}\right\}$ of lines defined as follows: 


$$
l(u, v)=\text { the unique line containing } \mathrm{p}\left[T^{\alpha}(u, v)\right]
$$

where $\alpha$ is the unique ordinal such that $\langle u, v\rangle \in T^{\alpha}-T^{\alpha+1}$. To see this, let $y$ witness that $x \in A=\mathrm{p}\left[T^{0}\right]$ and let $\alpha$ be a unique ordinal less than $\delta$ such that $\langle x, y\rangle \in\left[T^{\alpha}\right]-\left[T^{\alpha+1}\right]$. Here, the set $[T]$, the body of the tree $T$, is defined to be the set of all infinite paths through $T$. It follows that

$$
\langle x \uparrow n, y \uparrow n\rangle \in T^{\alpha}-T^{\alpha+1}
$$

for some $n \in \omega$. So we have $x \in l(x \uparrow n, y \uparrow n)$. The lines $l(u, v)$ are $L\left[T^{0}\right]$ definable uniformly in $\langle u, v\rangle$ since $T^{\alpha}$ are. Hence, in this case, $A$ can be covered by an $L\left[T^{0}\right]$-definable family of lines indexed by $T^{0}$ and we are done.

Case 2: $\quad T^{\delta} \neq \emptyset$.

In this case, by Lemma $3, \mathrm{p}\left[T^{\delta}\right]$ and hence $A$ contain a perfect subset with no three collinear points. Arguing in $L\left[T^{\circ}\right]$, we can find such a perfect set with a code in $L\left[T^{0}\right]$. This completes the proof of the theorem.

Corollary (of Theorem 1). If $\boldsymbol{\aleph}_{1}^{L[x]}$ is countable for all real numbers $x$, then every $\Sigma_{2}^{1}$ set on the plane which cannot be covered by a countable family of lines contains a perfect set with no three collinear points.

Proof. Since every $\Sigma_{2}^{1}(x)$ set is $\boldsymbol{K}_{1}$-Suslin via a tree on $\omega \times \omega_{1}$ which lies in $L[x]$, it can be covered by $L[x]$-definable lines if it does not contain a perfect set without collinear points. While $\aleph_{1}^{[[x]}$ is countable, there are only countably many $L[x]$-definable lines.

The converse of this corollary is also true as they noted in [EKM]. We also note here that, assuming $\boldsymbol{\aleph}_{1}^{L}$ is countable, the union of all $L$ definable lines is the largest $\Sigma_{2}^{1}$ set which does not contain any perfect subset without collinear points. As we will see in the next section, such a largest set among $\Sigma_{1}^{1}$ sets does not exist.

\section{§2. Effective contents of the theorem of van Engelen, Kunen, and Miller}

A well-known theorem on (lightface) $\Sigma_{1}^{1}$ set of reals says that, for each $\Sigma_{1}^{1}$ set $A \subseteq \mathbb{R}$, either $A$ is contained in the class of $\Delta_{1}^{1}$ reals or else there is a perfect subset of $A$ (see $4 \mathrm{~F} .1$ of [Mo]). For the theorem of van Engelen et al., the situation is quite similar. In fact, we have the following 
effective refinement of their result.

Theorem 2. Let $A$ be a $\Sigma_{1}^{1}$ set on the plane. If $A$ cannot be covered by $\Delta_{1}^{1}$ lines, then $A$ contains a perfect set with no three collinear points. We can find such a perfect set with code recursive in Kleene's $\mathcal{O}$, the hyperjump of the empty set.

Proof. Let $A$ be a $\Sigma_{1}^{1}$ set on the plane which cannot be covered by $\Delta_{1}^{1}$ lines. We may assume $A$ does not meet any $\Delta_{1}^{1}$ line at all.

Let $T$ be a recursive tree on $\omega \times \omega$ which projects onto $A$. Define,

$$
T^{*}=\left\{\langle u, v\rangle \in T \mid(\exists x, y)(\forall n)\left[\left\langle u^{\wedge}(x \uparrow n), v^{\wedge}(y \uparrow n)\right\rangle \in T\right]\right\} .
$$

We claim that for every $\langle u, v\rangle$ in $T^{*}, \mathrm{p}\left[T^{*}(u, v)\right]$ is not contained in a line. To see this, suppose contrary that there is a line $l$ which contains $\mathrm{p}\left[T^{*}(u, v)\right]$ for some $\langle u, v\rangle$ in $T^{*}$.

The set $\mathrm{p}\left[T^{*}(u, v)\right]$ is equal to $\mathrm{p}[T(u, v)]$, and this is a nonempty $\Sigma_{1}^{1}$ set without $\Delta_{1}^{1}$ element. It follows that $\mathrm{p}\left[T^{*}(u, v)\right]$ contains at least two elements. Thus, we have

$$
\begin{aligned}
x \in l & \Longleftrightarrow(\forall y, z)[y, z \in \mathrm{p}[T(u, v)] \& y \neq z \Longrightarrow x \in \overline{y z}] \\
& \Longleftrightarrow(\exists y, z)[y, z \in \mathrm{p}[T(u, v)] \& y \neq z \& x \in \overline{y z}] .
\end{aligned}
$$

This gives a $\Delta_{1}^{1}$ definition of $l$. A contradiction follows since we are assuming $A$ does not meet any $\Delta_{1}^{1}$ line. So, our claim is proved.

By Lemma 3 of Section $1, \mathrm{p}\left[T^{*}\right]$ contains a perfect set with no three collinear points. The set of all perfect trees $S$ such that $[S] \subseteq A$ and that $[S]$ does not meet any line more than two points is a nonempty $\Sigma_{1}^{1}$ set. So, by the Kleene basis theorem, we can find such a perfect subset of $A$ which has a code recursive in $\mathcal{O}$.

Corollary (of Theorem 2). Among $\Sigma_{1}^{1}$ sets which do not contain any perfect set without three collinear points, there is no largest one.

Proof. Clearly, each $\Delta_{1}^{1}$ line is a $\Sigma_{1}^{1}$ set, while the union of all such lines is not. To see that the union of all $\Delta_{1}^{1}$ lines is not a $\Sigma_{1}^{1}$ set, consider the proposition stated below.

Proposition. Let $C$ be a $\Pi_{1}^{1}$ set on the plane. If $C$ has positive plane measure, then for some $\Delta_{1}^{1}$ real $x$, the section $C_{x}$ has positive linear measure. Thus, every plane $\Pi_{1}^{1}$ set with positive measure meets a $\Delta_{1}^{1}$ line at uncountably many points. 
Proof. By a theorem of M. Kondô and T. Tugué, the set

$$
E=\left\{x \in \mathbb{R} \mid \text { the section } C_{x} \text { has positive measure }\right\}
$$

is $\Pi_{1}^{1}$. By the Fubini theorem, $E$ has positive linear measure if $C$ has positive plane measure. Being a $\Pi_{1}^{1}$ set of positive measure, $E$ must contain a $\Delta_{1}^{1}$ real by $H$. Tanaka's measure theoretical basis theorem [Ta].

In order to establish complete analogy between effective perfect set theorems and effective refinements of the result of van Engelen et al., we have to consider two more examples.

QuEsTION. Are there sets with the following properties?

(1) Largest set among $\Pi_{1}^{1}$ sets which do not contain perfect subsets with no three collinear points.

(2) $\Pi_{1}^{0}$ set which does not meet any $\Delta_{1}^{1}$ line at all.

\section{§3. A result on the Solovay model}

Let $M$ be a countable standard model of ZFC, the Zermelo-Fraenkel set theory with the axiom of choice, and let $\kappa$ be an inaccessible cardinal in the model $M$. A condition of the Lévy forcing is a function $p$ on the finite subset of $\kappa \times \omega$ into $\kappa$ such that whenever $(\alpha, n) \in \operatorname{dom}(p)$. we have $p(\alpha, n)<\alpha$. Let $\mathbb{P}$ be the set of all conditions of the Lévy forcing. If we let $\mathbb{P}_{\xi}$ denote the set of conditions $p$ such that $\operatorname{dom}(p) \subseteq \xi \times \omega$, then we have $\mathbb{P}=\bigcup_{\xi<\kappa} \mathbb{P}$.

In the rest of this paper, $G$ denotes a fixed $\mathbb{P}$-generic filter over $M$. And $M^{1}$ denotes the generic extension $M[G]$ of $M$ by $G$. The Solovay model $N$ is defined to be $\left(\mathrm{HOD}\left({ }^{\omega} \mathrm{Ord}\right)\right)^{M 1}$. This model is defined by R. M. Solovay [So]. The following proposition is well-known.

Proposition (See [So] or [Je] for details.).

(a1) $M^{1}$ is a model of ZFC and the continuum hypothesis.

(a2) $N$ is a model of $\mathrm{ZF}+\mathrm{DC}$, where $\mathrm{DC}$ denotes the principle of dependent choice.

(b1) In $M^{1}$, every set of reals definable from a countable sequence of ordinals is Lebesgue measurable, has the Baire property, and either is countable or contains a perfect subset.

(b2) In $N$, the same is true for an arbitrary set of reals.

Our goal in this section is to prove the following theorem. 
THEOREM 3.

(c1) In $M^{1}$, every set of reals definable from a countable sequence of ordinals either can be covered by a countable family of lines or else contains a perfect set with no three collinear points.

(c2) In $N$, the same is true for an arbitrary set of reals.

This answers a question in [EKM].

Since $N$ is countably closed in $M^{1}$, (c2) immediately follows from (c1). For a proof of (c1), the next lemma allows us to concentrate on sets on the plane which is definable over $M$, the ground model.

LEMMA 1 ("An important lemma" in [So]). Let $s$ be a countable sequence of ordinals in $M^{1}$. Then, in $M^{1}$, there is a $\mathbb{P}$-generic filter $H$ over $M[s]$ such that $M^{1}=M[s][H]$.

In short, this lemma says that if $M^{1} / M$ is a Lévy extension, so is $M / M[s]$. For some proofs of Lemma 1, see [So] or Exercise 25.11 of [Je].

In $M^{1}$, each $\mathbb{P}_{\xi}(\xi<\kappa)$ is countable and there are only countably many $\mathbb{P}_{\xi}$-dense subsets which lie in $M$. So, in $M^{1}$, there are many $\mathbb{P}_{\xi^{-}}$ generic filters over $M$.

We say filters $F_{0}, \cdots, F_{n-1}$ on $\mathbb{P}_{\xi}$ are mutually generic over $M$ if each $F_{i}(i<n)$ is $\mathbb{P}_{\xi}$-generic over $M\left[F_{0}, \cdots, F_{i-1}, F_{i+1}, \cdots, F_{n-1}\right]$ or, equivalently, if the product $F_{0} \times \cdots \times F_{n-1}$ is $\left(\mathbb{P}_{\xi}\right)^{n}$-generic over $M$ in the sense of the product forcing. If we let $\mathscr{G}_{\xi}$ denote the space (in $M^{1}$ ) of all $\mathbb{P}_{\xi}$-generic filters over $M$ endowed with the canonical topology, then for each $n$, the set of $n$-tuples of mutually generic filters is a comeager subset of $\left(\mathscr{G}_{\xi}\right)^{n}$ in the sense of the product topology. (By "the canonical topology", we mean the topology generated by the sets of the form $\left\{F \in \mathscr{G}_{\xi} \mid p \in F\right\}$ where $p \in \mathbb{P}_{\xi}$.)

Note that, in $M^{1}, \mathscr{G}_{\xi}$ is a perfect Polish space, since there are only countably many conditions in $\mathbb{P}_{\xi}$ and countably many $\mathbb{P}$-dense sets which lie in $M$. So, one can prove the next lemma by an argument similar to the standard proof of the Baire category theorem together with the "split and shrink" method which we have already used in the proof of Lemma 3 of Section 1.

Lemma 2. Argue in $M^{1}$. For each $\xi<\kappa$, there is a set $\mathscr{F} \subseteq \mathscr{G}_{\xi}$ with the following property: $\mathscr{F}$ is homeomorphic to the Cantor space ${ }^{\circ} 2$ and every distinct three elements of $\mathscr{F}$ are mutually generic over $M$. Moreover, 
we can find such an $\mathscr{F}$ as a subset of $\left\{F \in \mathscr{G}_{\xi} \mid p \in F\right\}$ where $p$ is a given condition in $\mathbb{P}_{\xi}$.

The following lemma is a key to our proof.

Lemma 3. Fix a $\xi<\kappa$. Let $\dot{a} \in M^{\mathrm{P}_{\hat{\xi}}}$ be a name of a point on the plane. And let $F_{0}, F_{1}$, and $F_{2}$ be filters on $\mathbb{P}_{\xi}$ which are mutually generic over $M$. If interpretations $\dot{a}\left[F_{0}\right], \dot{a}\left[F_{1}\right]$, and $\dot{a}\left[F_{2}\right]$ are distinct and collinear, then the line determined by these points is M-definable.

Proof. Let $l$ be the line passing through $\dot{a}\left[F_{0}\right], \dot{a}\left[F_{1}\right]$, and $\dot{a}\left[F_{2}\right]$. Without loss of generality, we may assume the origin $o$ of the standard coordinate is not on $l$. Let $\boldsymbol{m}$ be the foot of the perpendicular drawn from $\boldsymbol{o}$ to $l$. Then, one can compute $\boldsymbol{m}$ from any two of the $\dot{a}\left[F_{0}\right], \dot{a}\left[F_{1}\right]$, and $\dot{a}\left[F_{2}\right]$. So, we have

$$
\boldsymbol{m} \in M\left[F_{0}, F_{1}\right] \cap M\left[F_{1}, F_{2}\right] \cap M\left[F_{2}, F_{0}\right]=M .
$$

The equality above follows from the fact that $F_{0}, F_{1}$, and $F_{2}$ are mutually generic over $M$. Since $\boldsymbol{m}$ and $\boldsymbol{o}$ are in $M$ and since $\boldsymbol{m}$ determines the line $l$ uniquely relative to $o$, this line $l$ is $M$-definable.

We shall now turn to the proof of Theorem 3 .

Proof of Theorem 3. We work in $M^{1}$. Let $A$ be a set on the plane which is definable from a countable sequence $s$ of ordinals. Then there are a formula $\varphi$ of set theory and a finite array $\vec{a}$ of parameters from $M[s]$ such that

$$
A=\left\{x \in \mathbb{R}^{2} \mid M[s][x] \vDash \varphi(x, \vec{a})\right\} .
$$

In [Je], a set of reals with such a representation is said to be Solovay over $M[s]$. See Lemma 42.10 of [Je] for Solovayness of a set definable from a countable sequence of oridinals. By Lemma 1, we may assume $A$ is a set on the plane which is Solovay over the ground model $M$. So, let

$$
A=\left\{x \in \mathbb{R}^{2} \mid M[x] \vDash \varphi(x, \vec{a})\right\}
$$

where $\vec{a} \in M$.

From now on, suppose that $A$ cannot be covered by any countable family of lines. Since there are only countably many $M$-definable lines, $A$ has an element which is not on any $M$-definable line. It follows that there is a $\xi<\kappa$ such that 


$$
M\left[G \cap \mathbb{P}_{\xi}\right] \vDash " A-\bigcup\{l \mid l \text { is an } M \text {-definable line }\} \neq \emptyset " .
$$

Pick a condition $p \in G \cap \mathbb{P}_{\xi}$ and a name $\dot{x} \in M^{\mathrm{P}_{\xi}}$ of a point on the plane such that

$$
p \vdash_{\mathbf{p}_{\xi}} \text { “ } M[\dot{x}] \vDash \varphi(\dot{x}, \vec{a}) \& \dot{x} \notin \bigcup\{l \mid l \text { is an } M \text {-definable line }\} ” .
$$

By Lemma 2 , there is a set $\mathscr{F}$ of filters on $\mathbb{P}_{\xi}$ such that the following are satisfied.

(1) For every $F \in \mathscr{F}, p \in F$.

(2) Every three distinct elements of $\mathscr{F}$ are mutually generic over $M$.

(3) $\mathscr{F}$ is homeomorphic to the Cantor space.

For each $\mathbb{P}_{\xi}$-generic filter $\mathscr{F}$ over $M$, let

$$
h(F)=\dot{x}[F] \text {. }
$$

So, $h$ is a function on $\mathscr{G}_{\xi}$ into $\mathbb{R}^{2}$. This $h$ is continuous because the interpretation $\dot{x}[F]$ is approximated arbitrarily close by conditions contained in $F$. Since $p$ forces that $\dot{x}$ is an element of $A, h(F) \in A$ holds for every $F \in \mathscr{F}$.

Let $F_{0}, F_{1}$, and $F_{2}$ be distinct elements of $\mathscr{F}$ and suppose that $h\left(F_{0}\right)$, $h\left(F_{1}\right)$, and $h\left(F_{2}\right)$ were collinear. Then, by Lemma 3 , these three points would be on an $M$-definable line. This is impossible because $p$ forces that $\dot{x}$ is not on any $M$-definable line.

It follows that the restriction of $h$ to $\mathscr{F}$ is a continuous injection mapping every three distinct elements of $\mathscr{F}$ to points in $A$ which are not

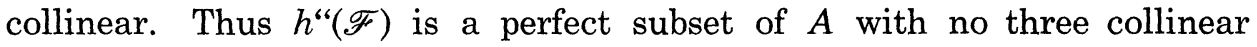
points.

The argument invoked in the above proof seems to work for establishing some homogeneity properties of open/closed partitions of pairs of reals as was considered by $\mathrm{Q}$. Feng in $[\mathrm{Fe}]$.

\section{REFERENCES}

[EKM] F. van Engelen, K. Kunen, A. W. Miller, Two remarks about analytic sets, Lecture Notes in Math., 1401 (1989), 68-72.

[Fe] Q. Feng, Combinatorial regularity properties of subsets of the real line, Proc. of 4th Asian Logic Conference, Tokyo (1990), 139-142.

[Je] T. Jech, Set Theory, Academic Press, London, 1978.

[Mo] Y. N. Moschovakis, Descriptive Set Theory, North-Holland, Amsterdam, 1980.

[So] R. M. Solovay, A model of set theory in which every set of reals is Lebesgue measurable, Ann. of Math., 92 (1970), 1-56. 
[Ta] H. Tanaka, A basis result for $\Pi_{1}^{1}$ sets of positive measure, Comment. Math. Univ. St. Paul, 16 (1968), 115-127.

Department of Mathematics

School of Science

Nagoya University

Chikusa-ku, Nagoya 464-01

Japan

Current address:

Department of Mathematics

Faculty of Science

Ehime University

Matsuyama 790

Japan 